Cahiers

Balkaniques

\section{Cahiers balkaniques}

Hors-série | 2015

Les élites grecques modernes, $\mathrm{XVIII} \mathrm{e}^{\mathrm{X}} \mathrm{XX}^{\mathrm{e}}$ siècles : identités, modes d'action, représentations

\title{
Élites éclairées et intellectuels progressistes grecs dans l'Alexandrie de Cavafy
}

Enlightened Elites and Progressive Intellectuals in Cavafy's Alexandria

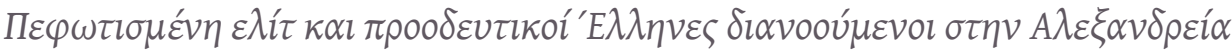

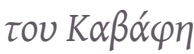

Sophie Coavoux

\section{OpenEdition}

Journals

Édition électronique

URL : https://journals.openedition.org/ceb/5565

DOI : 10.4000/ceb.5565

ISSN : 2261-4184

Éditeur

INALCO

Édition imprimée

Date de publication : 1 mars 2015

ISBN : 978-2-85831-224-5

ISSN : 0290-7402

\section{Référence électronique}

Sophie Coavoux, «Élites éclairées et intellectuels progressistes grecs dans l'Alexandrie de Cavafy », Cahiers balkaniques [En ligne], Hors-série | 2015, mis en ligne le 26 janvier 2016, consulté le 06 juillet 2021. URL : http://journals.openedition.org/ceb/5565; DOI : https://doi.org/10.4000/ceb.5565

Ce document a été généré automatiquement le 6 juillet 2021.

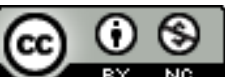

Cahiers balkaniques est mis à disposition selon les termes de la Licence Creative Commons Attribution - Pas d'Utilisation Commerciale 4.0 International. 


\section{Élites éclairées et intellectuels progressistes grecs dans l'Alexandrie de Cavafy}

Enlightened Elites and Progressive Intellectuals in Cavafy's Alexandria

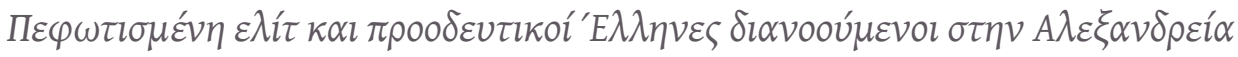

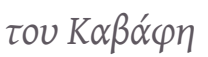

Sophie Coavoux

\section{Introduction}

1 Alexandrie, creuset cosmopolite et polyglotte, est, au début du $\mathrm{xx}^{\mathrm{e}}$ siècle, l'un des centres les plus prestigieux de l'hellénisme. Sous l'impulsion de Méhémet-Ali, fondateur de l'Égypte moderne, nombre de concessions sont cédées à des étrangers et on encourage en particulier le quasi-monopole grec de l'exportation. La plupart des Grecs sont arrivés en Égypte après 1840, pour échapper à l'étroitesse du nouvel État hellénique fondé en 1830, ou au joug ottoman qui pèse encore sur le reste de l'hellénisme, et pour se lancer dans le négoce et le commerce. Ils y parviennent brillamment et forment une sorte d'aristocratie très fortunée (les $\pi \rho \omega \tau o k \lambda \alpha \sigma \sigma \alpha \dot{\tau} \tau o)$. Ces Grecs des premières générations sont suivis par d'autres, qui constituent une nouvelle classe composée de petites gens et de bourgeois moyens, les $\delta \varepsilon v \tau \varepsilon \rho o k \lambda \alpha \sigma \sigma \alpha ́ \tau o l$, nouveaux riches incarnant la ploutocratie moderne.

2 Si les Grecs jouent un rôle prépondérant dans le négoce et le commerce, l'industrie et la banque, ils s'illustrent également dans le domaine des arts, des lettres et des idées. Les intellectuels grecs d'Alexandrie côtoient l'intelligentsia européenne et sont également en contact avec leurs homologues athéniens, participant activement, pour la plupart d'entre eux, aux débats politiques et culturels du centre national (notamment sur la question de la langue). 
Différentes tendances coexistent néanmoins au sein de cette élite et il existe un clivage entre deux groupes distincts. Les uns, issus de l'élite économique de la communauté égyptiote, œuvrent au maintien de la conscience nationale et des liens avec la métropole: les Bénakis, Salvagos, Kotzikas, etc., qu'Alexandre Kitroeff (1983, 20), reprenant un concept d'Antonio Gramsci (1983, 239 sqq), définit comme des « intellectuels organiques». Les autres, «intellectuels de gauche " pourrait-on dire aujourd'hui, sont en contact avec les cercles socialistes, internationalistes, syndicalistes ou anarchistes: Georges Vrissimitzakis, Sakellarios Yannakakis, Georgios Skliros, Stéphanos Pargas pour n'en citer que quelques-uns. Ces derniers se caractérisent par une propension certaine à l'avant-garde et à certaines tendances progressistes, sur le plan philosophique, esthétique, idéologique ou politique.

Il convient de se demander quelles sont les spécificités de ces différentes élites grecques. Présentent-elles des particularités et une identité propre par rapport à celles de la métropole? Quels sont leurs rapports avec leurs homologues de Grèce, d'Europe, ou encore d'Égypte? Quelle fut leur contribution à la construction de l'hellénisme moderne?

\section{Limites chronologiques}

5 La période qui nous intéresse ici s'étend environ des années 1900 aux années 1930 et

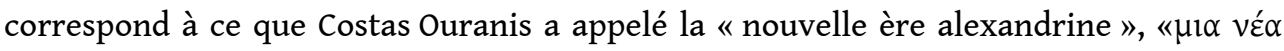

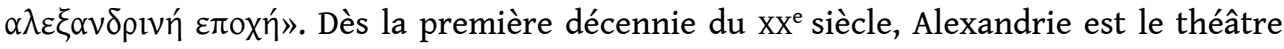
d'une vie intellectuelle florissante, à laquelle participent ardemment les intellectuels grecs, avec notamment l'activité nouvelle des revues.

6 Les témoignages abondent sur l'effervescence intellectuelle que connaît alors Alexandrie, qui, du point de vue grec, est souvent décrite comme le centre par excellence des lettres helléniques, qui s'impose face à Athènes. Pour Costas OURANIs (1955, 147-148), Giagos Iliadis (PIÉRIDIS, 1971, 12), mais aussi Georges Séféris (SARÉYANNIS, 1994, 9), ou Nikos Kazantzaki ${ }^{1}$, par exemple, Alexandrie apparait comme la rivale d'Athènes². On peut considérer 1904, année de création de la revue Néa Zoï, comme la date inaugurale de cette période. Quant à la fin de cette "nouvelle ère alexandrine ", Yannis Saréyannis la fait coïncider avec la mort de Cavafy³.

7 Figure centrale des cercles intellectuels alexandrins de l'époque, autour de laquelle gravite une communauté variée de l'intelligentsia européenne, Cavafy est en effet souvent perçu comme le symbole de cette période marquée par l'épanouissement de la vie intellectuelle. En outre, il est en quelque sorte le catalyseur qui se situe à la croisée des différentes tendances de l'intelligentsia grecque d'Alexandrie. Sans être au centre du présent exposé, il est ici évoqué comme simple point de référence.

\section{Le contexte historique}

8 Avant d'esquisser les différents profils de l'élite alexandrine, il convient de revenir sur le contexte historique général. À quoi ressemblait l'Alexandrie contemporaine de Cavafy (RISVA, 1981, 20-28 ; CHATZIPHOTIS, 1999 ; HAAG, 2005) ? Au début du Xx siècle, alors qu'Athènes ne compte environ que 170000 habitants - et, d'après le Service des statistiques de Grèce, si l'on inclut le Pirée et Kallithéa, 242328 âmes en tout - 
Alexandrie a une population de 400000 personnes, dont 90000 étrangers, membres des communautés grecque, italienne, allemande, anglaise, française, autrichienne, suisse, auxquels il faut ajouter les Syriens, les Arméniens et les ressortissants de divers autres pays d'Europe et d'Asie, formant avec les autochtones une société cosmopolite. La communauté grecque est l'une des plus importantes (снатzірнотіs, 1999, 70): le recensement officiel de 1917 indique la présence à Alexandrie de 25393 Grecs contre 36882 en 1937, chiffres sans doute en deçà de la réalité puisque n'étaient recensés que les Grecs de nationalité hellénique et non, par exemple, les sujets ottomans ${ }^{4}$. Mais c'est la communauté anglaise qui domine puisque, à la suite du bombardement d'Alexandrie par leur flotte en juillet 1882, l'Égypte est occupée militairement par les Britanniques, puis traitée par ces derniers en colonie, jusqu'en $1914^{5}$.

9 La paroikia d'Alexandrie se caractérise par une composition sociale variée. En effet, outre les $\pi \rho \omega \tau \sigma \kappa \lambda \alpha \sigma \sigma \alpha \dot{z} \tau o l$ et les $\delta \varepsilon v \tau \varepsilon \rho o \kappa \lambda \alpha \sigma \sigma \alpha ́ \tau o l$, se rencontrent également nombre de petites gens, ouvriers (ouvriers spécialisés), petits commerçants, employés de bureaux ou garçons de café.

10 La paroikia trouve sa cohérence dans divers points d'ancrage qui assurent des liens forts entre ses membres, comme l'Église grecque orthodoxe, le Consulat (proxenio), la presse grecque locale, les nombreuses associations et autres organisations communautaires. Le point de référence central est sans aucun doute la koinotita, organe collectif administré par les élites économiques de la communauté (souloyAnNIS, 1994). La koinotita assure et surtout finance de nombreuses activités essentielles à la paroikia qu'elle dote d'écoles, de fondations de bienfaisance, d'églises, d'une maison de retraite et d'associations sportives et culturelles. Le niveau moyen d'instruction des Grecs d'Alexandrie est alors supérieur à celui des Grecs de la métropole, comme l'atteste notamment le nombre de diplômés d'université (TRIMI-YANNAKAKIS, 1992, 84). En outre, la vie culturelle de la paroikia est intense: expositions, théâtre, revues, quotidiens, maisons d'édition, librairies.

11 La koinotita se trouve sous l'influence directe de la ploutocratie qui lui assure un soutien financier, et dépend par ailleurs des autorités helléniques (TRIMI KIROU, 2003, 4). Elle joue un rôle de tout premier plan dans le maintien de la conscience nationale, dans l'identification à l'État-nation hellénique et l'idéologie nationaliste (TRIMI KIROU, 2003, 4). L'attachement des Égyptiotes à la patrie est d'ailleurs attesté par divers indicateurs comme leur participation aux différentes luttes nationales, notamment aux guerres balkaniques (souloyanNIs, 1994, 278), leur implication dans les questions nationales (toutes relayées par la presse locale grecque), ou encore les aides financières prodiguées à la Grèce. En outre, dès la fin du xix $x^{e}$ siècle, les liens de plus en plus étroits de la koinotita avec la Grèce sont surtout visibles dans le domaine de la politique éducative et dans les programmes scolaires (TRIMI KIROU, 2003, 4) ${ }^{6}$.

\section{L'élite économique}

Venons-en à présent à l'élite économique qui, établie à Alexandrie depuis la moitié du $\mathrm{XIX}^{\mathrm{e}}$ siècle, a prospéré économiquement et bâti de grandes fortunes. Les Bénakis en sont l'un des meilleurs exemples. De fait, cette élite économique est composée d'hommes d'affaires modernes qui perpétuent la tradition de l'évergétisme tant en Égypte qu'en Grèce ${ }^{7}$. Selon Ilios Yannakakis (YANNAKAKIS, 1992, 134 sqq) : 
Le cosmopolitisme alexandrin possède une histoire propre, singulière. Il plonge ses racines dans l'évergétisme des élites communautaires. Installées à Alexandrie depuis les années 1940 et 1950 du XIX siècle, ces élites ont prospéré économiquement et sont à la tête d'immenses fortunes (les Gianaklis, Salvago, Kotzikas, Benaki, Menasce, Matoussian, etc.). Elles perpétuent, en l'amplifiant, la tradition de "bienfaisance», telle qu'elle existait dans les millets de l'Empire ottoman. Entrepreneurs modernes, cultivés, inspirés par le progrès social de l'Europe de la seconde moitié du XIXe siècle, ces élites dotent leurs communautés respectives d'institutions philanthropiques et culturelles au service de leurs compatriotes immigrés.

13 Caste économique éclairée, ils prennent part à la vie intellectuelle alexandrine. "Le cercle international animé par l'“ami des arts", l'Arménien Sarkissian, et les Grecs Bénakis et Nomikos, invite régulièrement pour des conférences des célébrités européennes [...] » (YANNAKAKIS, 1992, 139). Antonis Bénakis, à l'instar d'autres hommes de son rang, constitue sa collection privée d'objets d'art. Les Salvagos tiennent salon, animé par Argini Bénakis Salvagos, fille d'Emmanuel Bénakis (TSIRKAS, 1978, 20).

Outre cette activité intense sur le plan culturel et intellectuel, ce qui caractérise sans doute le mieux l'esprit de ces élites et le sens de leur action, c'est leur lien avec la Grèce et l'idéologie nationaliste qui l'accompagne, dont ils se font le relais auprès des Égyptiotes. Emmanuel Bénakis incarne parfaitement cette caste. Il compte parmi les plus riches Grecs d'Alexandrie et de l'ensemble de la diaspora. Sur le plan économique, ses actions de soutien à la Grèce sont bien connues (on pense notamment au don qu'il fit à la Banque nationale de Grèce). Sur le plan politique, fervent vénizéliste, son engagement le pousse à entrer en politique (il s'établit à Athènes) ${ }^{8}$. Citons également l'exemple de Mikès K. Salvagos, président de la Communauté grecque de 1919 à 1948, lui aussi vénizéliste ${ }^{9}$. De fait, auprès de ces élites bourgeoises éclairées (comme d'ailleurs auprès de la paroikia en général), Elefthérios Venizélos a trouvé un soutien indéfectible à sa politique libérale au service de la Grande Idée.

Cette élite se caractérise donc avant tout par un sentiment national fervent, et par son rôle sur le plan non seulement économique, mais également social, politique et intellectuel (TOMARA, 2006). Par leur attachement à la mère patrie, ces «intellectuels organiques » semblent représenter un modèle helléno-centriste, nationaliste, qu'on pourrait qualifier d'helladique. Ils incarnent l'Alexandrie décrite par Séféris comme un «melting-pot de cultures héritées des Lumières, fondées sur les valeurs d'une bourgeoisie progressiste ».

\section{L'élite intellectuelle et la gauche}

Aux côtés de ces élites issues de la ploutocratie locale, de ces «intellectuels organiques ", d'autres cercles de l'intelligentsia grecque alexandrine se développent, avec des orientations différentes. Cette deuxième grande tendance, que nous croyons pouvoir discerner dans l'élite grecque alexandrine de l'époque, est protéiforme, puisque les limites entre les diverses expressions de la gauche sont fluctuantes. Elle regroupe des intellectuels influencés, à divers degrés, par des idées radicales de gauche (principes socialistes et anarchistes), qui furent introduites dès la fin du XIx ${ }^{e}$ siècle dans les villes de Méditerranée orientale et plus spécifiquement Le Caire et Alexandrie (KHURI-MAKDISI, 2008). Ce milieu intellectuel - qui côtoie parfois le milieu syndicaliste ou se confond avec lui - est absolument cosmopolite. Pour une part, il est plus proche des 
cercles littéraires - et ce phénomène est sans doute moins connu, alors qu'il explique, pour une large part, la dimension avant-gardiste d'une partie de la littérature grecque alexandrine. L'Alexandrie du début du $\mathrm{xx}^{\mathrm{e}}$ siècle permet en effet la rencontre de nombreux intellectuels et hommes de lettres européens, devenant en quelque sorte un centre névralgique dans l'histoire européenne des idées: E. M. Forster, le futuriste Filippe Tommaso Marinetti, Enrico Pea, Guiseppe Ungaretti, Atanasio Catraro ; poètes, hommes de lettres, artistes (français, italiens, grecs) se retrouvent, à l'ouest d'Alexandrie, dans la fameuse maison du Mex des frères Thuile (Henri, le poète, et JeanLéon, le romancier), «le laboratoire littéraire le plus actif d'Alexandrie » (BASCH, 2004, 76), mais également à la Baracca Rossa, lieu de rencontre des artistes et des anarchistes, fondé par l'écrivain italien Enrico Pea (1881-1958), ou encore chez Constantin Cavafy. Il faut noter que ces intellectuels européens n'évoluent pas totalement en vase clos mais sont en contact, pour certains du moins, avec leurs homologues arabophones ${ }^{10}$.

Par le biais de ces milieux intellectuels cosmopolites, «[...] des idées révolutionnaires européennes pénètrent Alexandrie et de là se diffusent dans toute l'Égypte. Les Italiens introduisent l'anarcho-syndicalisme et la libre pensée, les Arméniens le corporatisme artisanal, les Grecs le syndicalisme, les Juifs le communisme. » (YANNAKAKIS, 1992, 136).

Les intellectuels grecs notamment se montrent très actifs dans la reprise et la diffusion de ces idées de gauche, sous leurs expressions diverses (anarcho-individualiste, anarcho-syndicaliste, marxiste et internationaliste). On voit se multiplier revues, publications $^{11}$, associations et autres projets de coloration anarchiste, comme l'Université populaire libre en 1901 (GORMAN, 2008).

19 S'ils ne s'illustrent pas nécessairement dans l'action politique, ces intellectuels grecs véhiculent des idées progressistes : appel à la justice sociale, égalité, éducation laïque et de masse, le tout sur fond de récusation de l'ordre social, politique et moral (KHURIMAKDISI, 2008). Principaux vecteurs de cette effervescence intellectuelle, les nombreuses revues $^{12}$ littéraires grecques de haut niveau créées dès les années 1900 : Néa Zoï (1904-1918) puis (1922-1928), Sérapion (1909-1910), Grammata ${ }^{13}$ (1911-1921), o Phoinikas (1916), Ta Propylaia (1916), Argo (1923-1927), Alexandrini Techni (1926-1930), etc. Notamment, les équipes des revues d'avant-garde Néa Zoï et Grammata redécouvrent Giorgos Skliros ${ }^{14}$, sociologue marxiste, auteur de la Question sociale, érigé en figure intellectuelle centrale de la paroikia, et organisent des conférences. Giorgos Skliros continuera par la suite à donner des conférences dans le cadre de l'Endefktirio du Caire, une association visant à la diffusion d'idées progressistes, fondée en 1915 à son initiative et à celle de Giorgos Pétridis, qui partageait ses idées (TRIMI KIROU, 2008, 4-5).

À noter que d'autres associations similaires voient le jour, à Alexandrie, au Caire et à Port-Saïd, fondées par des intellectuels grecs (ce sont souvent les mêmes noms qui reviennent: Giorgos Pétridis, Giorgos Skliros, Iordanis Iordanidis, Polys Modinos, Stéphanos Pargas, Sakellarios Yannakakis): les deux succursales du Cercle Éducatif (Ekpedeftikos Omilos, Alexandrie et Le Caire), l'Endefktirio de Port-Saïd, Omilos Kinonikon Meleton (Cercle des études sociales, Alexandrie, 1920) et Dimotistiki Omada.

Par parenthèse, il faut rappeler que Grammata ne se caractérise pas uniquement par sa coloration libertaire mais également, et cela est complémentaire, par une orientation clairement hédoniste - Cavafy y publiera pour la première fois ses poèmes érotiques. C'est aussi dans Grammata que Georges Vrissimitzakis, fondateur du groupe anarchisant des Apouanistes (1915), publie son fascicule consacré à l'Alexandrin, en qui il trouve un 
écho des aspirations et des idéaux apouanistes. Après avoir étudié à Paris, en 1911-1912, les lettres et la sociologie, Georges Vrissimitzakis passe une année en Italie où il entre en contact avec le cercle artistique à tendance anarchiste des Apouanistes à la tête duquel se trouve alors le peintre Lorenzo Viani, groupe anarchiste et socialiste qui compte également Enrico Pea (qui connut Cavafy même si ce dernier ne semble pas avoir fréquenté la Baracca Rosa). De retour à Alexandrie, toujours à la recherche de la

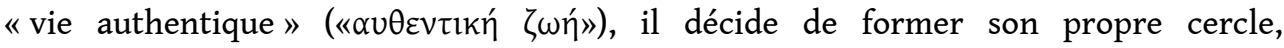
révolutionnaire et artistique, et, en 1915, réunit autour de lui de jeunes Alexandrins parmi lesquels on compte notamment Vassilis Athanassopoulos, Pétros Alitis, Nikos Santorinios, Sakellarios Yannakakis, Panos Kornessis. Georges Vrissimitzakis publie également, à l'instar d'autres Grecs $^{15}$, des textes d'inspiration anarchiste, comme I Atomiki Epanastasi (1914), ainsi qu'une traduction de Mikhaïl Bakounine ${ }^{16}$.

Parallèlement aux idées théoriques et philosophiques qu'ils développent, ces intellectuels de gauche prennent part au débat sur la question de la langue et, vraisemblablement sous l'influence de Giorgos Skliros, se révèlent d'ardents partisans du démoticisme. Ils participent ainsi à la fondation du Groupe Démoticiste (Dimotistiki Omada), et d'autres cercles, cités précédemment, sont également impliqués dans cet engagement. Sur ce point, ils rejoignent donc les idées des « intellectuels organiques ».

\section{Conclusion}

Il faut souligner qu'il serait hasardeux d'affirmer, selon une logique binaire, que ces deux types d'élites sont absolument imperméables. En effet, outre la question de la langue, il existe des exemples de passerelles entre les deux groupes: Cavafy, qui fréquente aussi bien les Bénakis, Antonis et Pénélopi Delta, que Skliros ou les Apouanistes, en est une parfaite illustration. Autre exemple: Giorgos Skliros qui rencontre Elefthérios Venizélos lors de sa visite à Alexandrie (HAAs, 2012). Enfin, dernier exemple, le Cercle Éducatif (Ekpedeftikos Omilos) d'Alexandrie rassemble des personnalités appartenant aux deux groupes: Antonis Bénakis mais aussi Giorgos Skliros, Stéphanos Pargas (снатziphotis, 1999, 172).

Et c'est précisément ce point qui est intéressant : les deux profils de l'élite alexandrine de l'époque, malgré des sensibilités a priori antagonistes, trouvent des points de convergence sur certains sujets, à commencer par la question de la langue.

Par leur attachement à la mère patrie, et dans le souci de protéger leurs intérêts, les «intellectuels organiques" semblent donc incarner, nous l'avons dit, un modèle helléno-centriste, nationaliste, qu'on pourrait qualifier d'helladique. Quant aux intellectuels de gauche, s'ils ne veulent pas complètement tourner le dos à la mère patrie, loin d'adopter une posture strictement helléno-centriste, ils optent pour une vision panhellénique, cosmopolite, plus européenne. Il faut retenir leur contribution significative par rapport au nationalisme et à l'idéologie colonialiste (GORMAN, 2008), résultat de leur contact avec les cercles socialistes, internationalistes, syndicalistes ou anarchistes.

Pour conclure et malgré tout, les deux tendances de l'élite alexandrine de la période considérée peuvent, à certains égards, être qualifiées de progressistes et toutes deux se sont distinguées par leur apport majeur à l'hellénisme moderne. 


\section{BIBLIOGRAPHIE}

BASCH Sophie, 2004, les Sublimes Portes. D'Alexandrie à Venise, parcours littéraire dans l'Orient fin de siècle, Paris : Honoré CHAMPION.

CATTAUI Georges, 1964, Constantin Cavafy, Paris : Seghers.

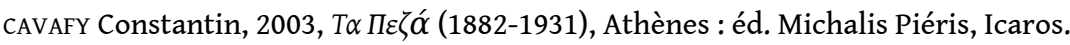

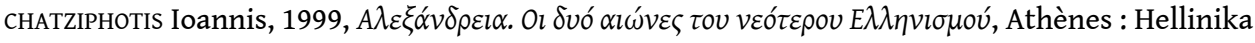
Grammata.

GORMAN Anthony, 2008, " Socialisme en Égypte avant la Première Guerre mondiale : la contribution des anarchistes ", Cahiers d'histoire, Revue d'histoire critique [En ligne], http:// chrhc.revues.org/1241, consulté le 7 octobre 2015.

GORMAN Anthony, 2009, "Repatriation, Migration or Readjustment: Egyptian Greek Dilemmas of the 1950s", in Greek Diaspora and Migration since 1700: Society, Politics and Culture, Ashgate: Edited by Dimitris TZIovAs, Farnham.

GRAMSCI Antonio, 1983, Textes (1917-1934), les Cahiers de la prison, 12, Paris : Éditions sociales.

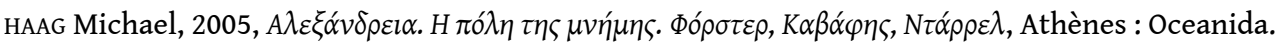

HAAS Diana, 2012, “Cavafy, Venizelos, and the National Schism: Revisiting a Debate", in Historical Poetics in Nineteenth and Twentieth Century Greece: Essays in Honor of Lily Macrakis, ed. Stamatia Dova. Classics @: An Online Journal (Center for Hellenic Studies, Harvard University), issue 10, [En ligne], http://chs.harvard.edu/wa/pageR?tn=ArticleWrapper\&bdc=12\&mn=4879, consulté le 7 octobre 2015.

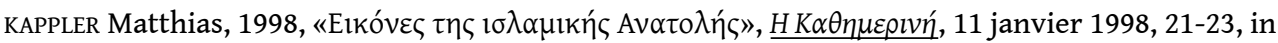
http://documents.tips/documents/kathimerini.html, consulté le 7 octobre 2015.

KAZAMIAS Alexander, 2009, "Between Language, Land and Empire: Humanist and Orientalist Perspectives on Egyptian-Greek Identity", in Greek Diaspora and Migration since 1700: Society, Politics and Culture, Ashgate: Edited by Dimitris Tziovas, Farnham.

KITROEFF Alexander, 1983, "The Alexandria we have lost". Journal of the Hellenic Diaspora, vol. x, $\mathrm{n}^{\circ} 1-2, \mathrm{p} \cdot 11-22$.

KHURI-MAKDISI Ilham, (2008), "Intellectuels, militants et travailleurs : la construction de la gauche en Égypte, 1870-1914 ». Cahiers d'histoire, Revue d'histoire critique, 105-106. [En ligne] http:// chrhc.revues.org/504, consulté le 7 octobre 2015.

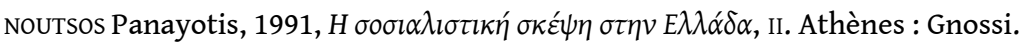

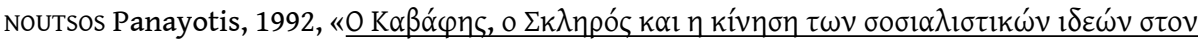

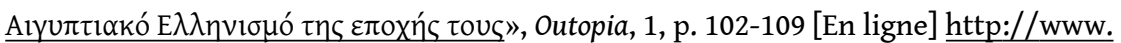
24grammata.com/wp-content/uploads/2012/05/Noutsos_24grammata.com_.pdf, consulté le 7 octobre 2015.

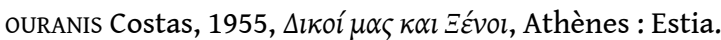

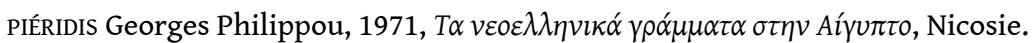


RISVA Marina, 1981, la Pensée politique de Constantin Cavafy, Paris : Les Belles Lettres, coll. Néohellénique.

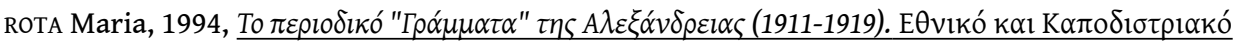

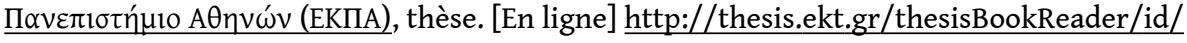
24684\#page/60/mode/1up, consulté le 7 octobre 2015.

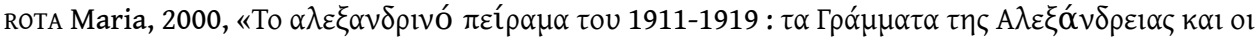

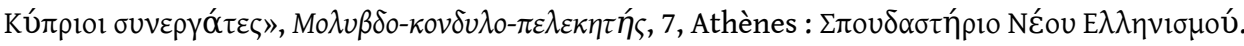

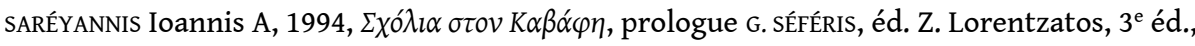
Athènes : Icaros.

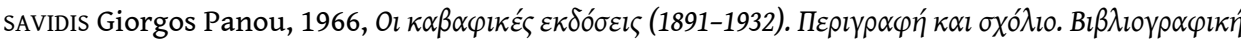

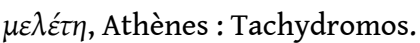

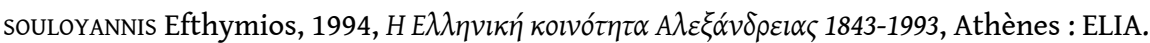

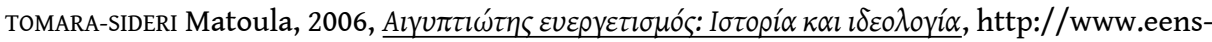
congress.eu/?

main__page=1\&main__lang=de\&eensCongress_cmd=showPaper\&eensCongress_id=128, consulté le 7 octobre 2015

TRIMI KIROU Katerina, 2003, « Quel cosmopolitisme à l'ère des nationalismes ? ", Cahiers de la Méditerranée, 67. [En ligne] http://cdlm.revues.org/130, consulté le 7 octobre 2015.

TRIMI KIROU Katerina, 1996, Kinotis Grecque d'Alexandrie: Sa politique éducative (1843-1932), thèse de doctorat, Université de Sciences humaines de Strasbourg.

TRIMI KIROU Katerina, YANNAKAKIS Ilios, (1992), « Les Grecs : la parikia d'Alexandrie », in Alexandrie 1860-1960. Un modèle de convivialité: communautés et identité cosmopolite, dirigé par Robert ILBERT et Ilios YANNAKAKIS, avec la collaboration de Jacques HASSOUN, Paris : éd. Autrement, série Mémoires, $\mathrm{n}^{\circ} 20$.

TRIMI KIROU Katerina, (2008), «Être internationaliste dans une société coloniale : le cas des Grecs de gauche en Égypte (1914-1960) ", Cahiers d'histoire, Revue d'histoire critique http:// chrhc.revues.org/513, consulté le 7 octobre 2015.

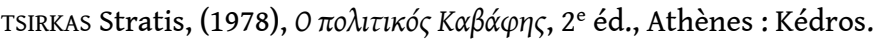

YANNAKAKIS Ilios, (1992), «Adieu Alexandrie! », in Alexandrie 1860-1960. Un modèle de convivialité: communautés et identité cosmopolite, dirigé par Robert ILBERT et Ilios YANNAKAKIS, avec la collaboration de Jacques HASSOUN, Paris : éd. Autrement.

\section{NOTES}

1. Kazantzaki, après avoir visité l'Égypte en 1927, écrit sur Cavafy et sur le cercle

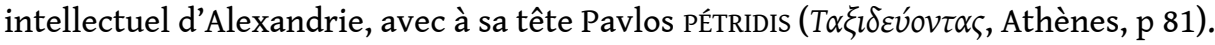

2. Sur les différentes perceptions de la littérature grecque d'Égypte, voir KAZAMIAS, 2009, 177-178. Également, ROTA, 2000, 146-147.

3. Concernant le début de cette période, Maria Rota propose quant à elle 1904 (ROTA, 1994). Pour ce qui est de la fin, voir SARÉYANNIS, 1994, 9. 
4. La question du dénombrement des Grecs d'Égypte est sujette à caution cf. GORMAN, 2009, 62 .

5. En droit, le pays fait partie intégrante de l'Empire ottoman, mais il est en réalité sous la domination anglaise.

6. Sur le sujet de l'enseignement, voir également TRIMI KIROU, 1996: elle signale notamment que le symbole le plus éloquent de l'orientation nationaliste de la koinotis est l'inscription gravée sur la façade d'un bâtiment scolaire : «Rien de plus sacré que la patrie».

7. Voir la liste établie par souloyannis $(1994,278)$. Voir également CHATZiphotis (1999, 142).

8. Sur le sujet, voir HAAG, 2005, 114 sqq.

9. TSIRKAS, 1978, 19-20.

10. Cavafy a notamment rencontré le Libanais Khalil Moutran par le truchement de Georges Cattaui. Mais contrairement à l'assertion de ce dernier (1964, 40), Cavafy a manifesté un intérêt réel pour la culture arabe et les hommes de lettres arabophones,

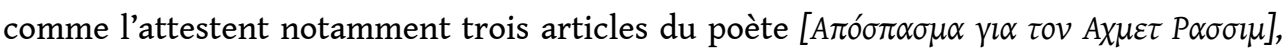

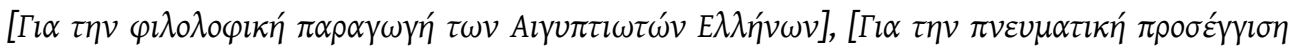
Alyúrtov $\Delta v ́ \sigma\rceil \zeta]$ (CAVAFY, 2003). Sur le sujet, voir également KAPPLER, 1998.

11. «Cette presse fut complétée par une littérature locale significative composée de pamphlets locaux et de livres, publiés dans les années précédant le premier conflit mondial, particulièrement par les anarchistes grecs. Cela reflétait les différents éléments à la fois du courant individualiste et de la tendance anarcho-syndicaliste. » (GORMAN, 2008).

12. D'ailleurs, ces revues fleurissent à un moment où les revues équivalentes qui paraissent à Athènes traversent une phase défavorable (ouRANIS, 1955, 147). Et l'une des raisons qui président à la fondation de la revue Grammata est de contredire l'idée reçue selon laquelle les hommes de lettres alexandrins de l'époque seraient inférieurs à leurs homologues athéniens (ROTA, 2000, 152).

13. La revue Grammata en particulier a joué un rôle de premier plan dans cette mouvance intellectuelle, en développant une philosophie progressiste à coloration anarchiste.

14. Cavafy fait la connaissance de Skliros en décembre 1913 (NOUTSOS, 1992).

15. Sur ces publications, voir NouTsos, 1991, 453-455.

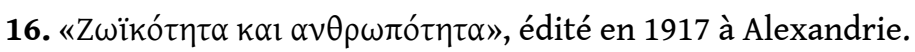

\section{RÉSUMÉS}

Alexandrie est, au début du $\mathrm{xx}^{\mathrm{e}}$ siècle, l'un des centres les plus prestigieux de l'hellénisme. Si les Grecs occupent une place prépondérante dans le négoce et le commerce, dans l'industrie et la banque, ils s'illustrent également dans le domaine des arts, des lettres et des idées. L'une des 
spécificités des élites grecques d'Alexandrie réside dans une propension à l'avant-garde et à certaines tendances progressistes, tant sur le plan esthétique qu'idéologique ou politique. Alors que les «intellectuels organiques» semblent incarner un modèle helléno-centriste et nationaliste, qu'on pourrait qualifier d'helladique, les intellectuels de gauche, pour leur part, loin d'adopter une posture strictement helléno-centriste, optent pour une vision panhellénique, cosmopolite, plus européenne.

In the early Twentieth century, Alexandria is one of the most prestigious centres of Hellenism. If the Greeks held a key role in trade and commerce, industry, banking, they also distinguished themselves in the realm of arts, literature and ideas. One of the specificities of Alexandrian Greek elites lies in a certain propensity to avant-garde and progressive trends, both aesthetic and ideological or political. On the one hand, the "organic intellectuals" seem to embody a Hellenocentric, nationalist, Helladic model. As for the left-wing intellectuals, far from adopting a strictly Hellenocentric posture, they opt for a more European, cosmopolitan, Panhellenic vision.

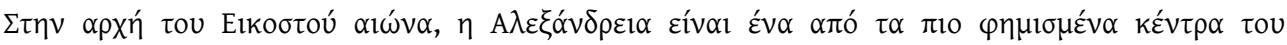

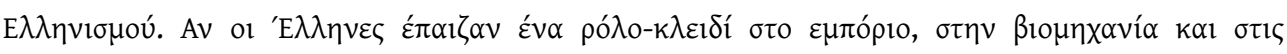

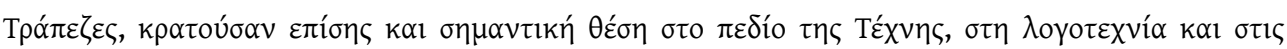

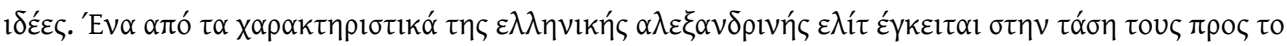

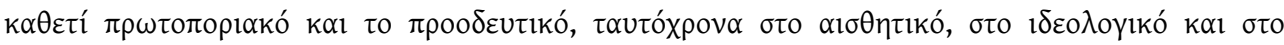

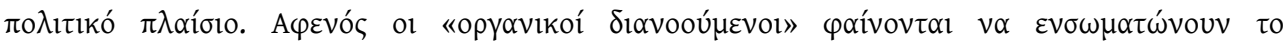

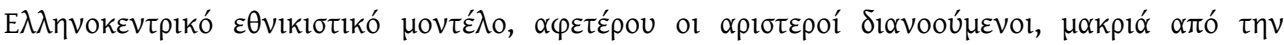

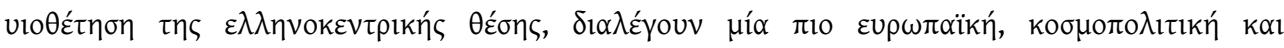

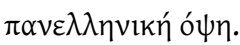

\section{INDEX}

motsclesmk КАВАФИС КОНСТАНТИН (1863-1933), ХЕЛЕНИЗМОТ, АЛЕКСАНДРИЈА, ПОЧЕТОКОТ НА ДВАЕСЕТТИОТ ВЕК, КУЛТУРНАТА ИСТОРИЈА

motsclestr Kavafis Konstantin (1863-1933), Helenizm, İskenderiye, Yirminci yüzyılın başlarında, Kültür tarihi

Index géographique : Alexandrie

Thèmes : Histoire culturelle

Mots-clés : Cavafis Constantin (1863-1933), Cavafis Constantin (1863-1933), hellénisme, Alexandrie

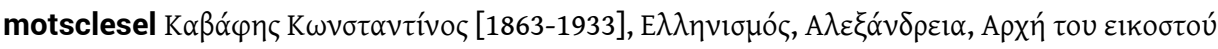

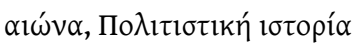

Keywords : Cavafis Constantine (1863-1933), Hellenism, Alexandria, Beginning of the Twentieth century, Cultural history

Index chronologique : vingtième siècle -- début

\section{AUTEUR}

\section{SOPHIE COAVOUX}

IETT, Université Jean Moulin Lyon 3 